\title{
How to Approach Delirium: A Case Report
}

Volume 2 Issue 3- 2021

\author{
Author Details \\ Sabrina Dar*, Diago Martinucci-Canto \\ St. Elizabeth's Medical Center, USA \\ *Corresponding author \\ Sabrina Dar, St. Elizabeth's Medical Center, Boston, USA \\ Article History \\ Received: August 03, 2021 Accepted: August 27, 2021 Published: August 30, 2021
}

\begin{abstract}
Throughout our clinical practice, we all have encountered delirium at some point in time and have been confused about what we should do. We as practitioners are naturally inclined to using high-dose antipsychotics for agitation which can increase allcause mortality [1]. Delirium is a hypocholinergic-hyperdopaminergic state [1]. This case report helps simplify the steps a provider should take in approaching Delirium to minimize all-cause mortality, for which FDA has issued a black box warning [1]. As Delirium can be seen in any hospital unit, all healthcare providers would benefit from this case report, so the primary team, in any setting, can handle this presentation appropriately in an acute situation. The first approach should be nonpharmacological. After exhausting nonpharmacological approaches, pharmacological approaches may be used, including low-dose antipsychotics, with a maximum daily dose of the lower limit of the therapeutic range of that antipsychotic [1]. Providers must be mindful that delirium may last indefinitely, and that medications discussed in this case report are not to cure, rather to manage the symptoms [1].
\end{abstract}

\section{Introduction}

Throughout our clinical practice, we all have encountered delirium at some point in time and have been confused about what we should do. We as practitioners are naturally inclined to using high-dose antipsychotics for agitation, however, is this really the right thing to do? NO! High-dose antipsychotics are the worst thing to do as it can increase all-cause mortality [1]. Let us first discuss what delirium is; a hypocholinergic-hyperdopaminergic state [1]. Per Diagnostic and Statistical Manual of Mental Disorders, Fifth Edition (DSM-5), it is a disturbance in attention and awareness from baseline, that can develop over a short period of time, fluctuate in severity during the course of a day, and there is evidence this presentation is a direct physiological consequence of another medical condition [2]. The three main types are hyperactive, hypoactive, and mixed [2]. Delirium hyperactive type is better known in practice due to the agitated and combative presentation. As opposed to delirium hypoactive type, as it is easily overlooked and misinterpreted as depression. The aim of this case report is to make aware of how to approach delirium with its various presentations, particularly the challenging hyperactive type.

\section{Case Description}

An 84-year-old male with a past diagnosis of Major Neurocognitive Disorder, Coronary Artery Disease, Hypertension, presented with behavioral disturbance. Five years ago, he was still active and managing his own bills. Since then, he has had a slow decline in cognition, and started going to daycare. Approximately one-year ago, he was placed in a nursing home $(\mathrm{NH})$ for assistance with activities of daily living (ADLs). Recently at the $\mathrm{NH}$, he went into someone else's room, and punched them because he misbelieved the resident stole his cell phone. He was then placed on a section 12, sent to a geriatric psychiatry unit (GPU) for behavioral disturbance. Upon arrival to the GPU, patient sustained a fall, and hit his head. Patient was transferred to the Intensive Care Unit (ICU) for evaluation by neurosurgery. While in the ICU, patient attempted to go to the bathroom, slipped on his urine, and sustained another fall, with head strike. Head CT was obtained (Figure 1), which showed a Left Temporal Subarachnoid Hemorrhage.

Neurosurgery signed off, as the bleed was stable. Patient continued to be agitated, combative, and disoriented. Primary team ordered olanzapine 10 milligrams (mg) intramuscular (IM). Then ordered an electrocardiogram (EKG) (Figure 2), which showed QTc prolongation.

They then discontinued the antipsychotic to avoid further QTc prolongation, and possible induction of torsades de pointes, and started Dexmedetomidine for agitation. Neurology and psychiatry were subsequently consulted for further assistance. Neurology diagnosed patient with acute delirium on chronic dementia secondary to subarachnoid hemorrhage (SAH). Psychiatry agreed and recommended nonpharmacological methods for reorientation, and Olanzapine $2.5 \mathrm{mg}$ IM twice daily as needed (Table 1), not to exceed $5 \mathrm{mg} /$ day for agitation [1]. Patient responded well and switched from delirium hyperactive to hypoactive. Once medically stable, he was discharged to a memory unit for further care. 


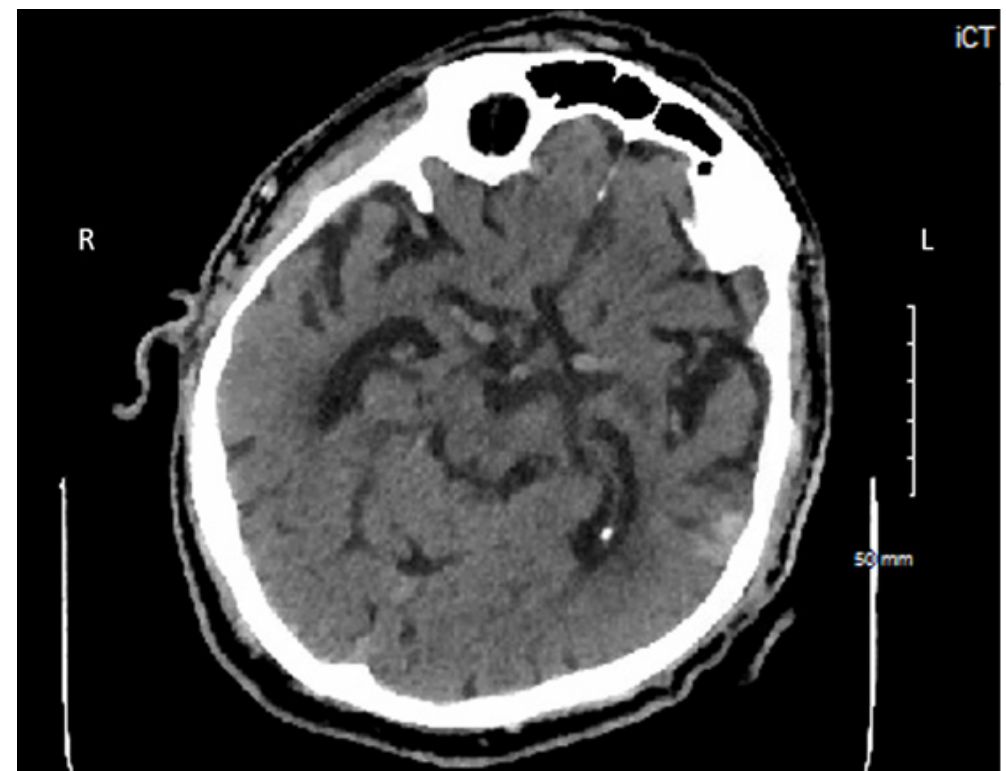

Figure 1: Head CT- Left Temporal Subarachnoid Hemorrhage.

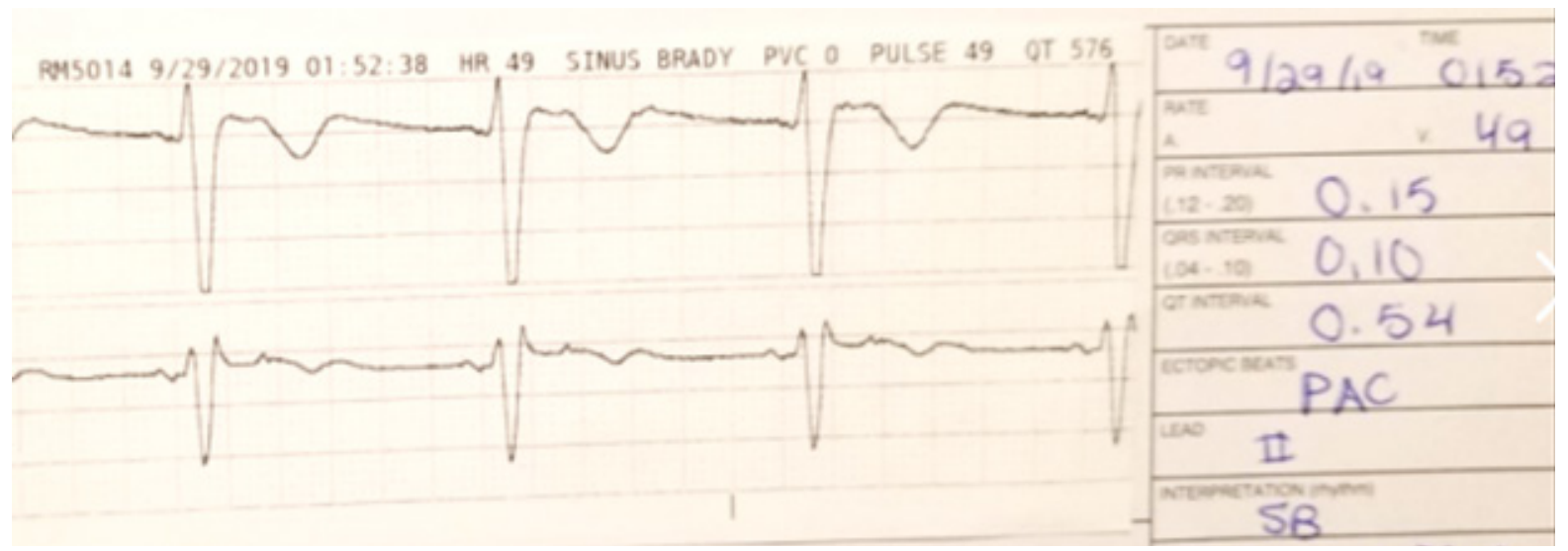

Figure 2: Electrocardiogram.

Table 1: Recommended pharmacological intervention for Delirium.

\begin{tabular}{|c|c|c|}
\hline Medication & Regimen & Route \\
\hline \multirow{2}{*}{ Haloperidol } & $0.5 \mathrm{mg}$ BID & \multirow{2}{*}{ PO, IV, IM } \\
\hline & $1 \mathrm{mg} \mathrm{HS} \mathrm{SCH}$ & \\
\hline \multirow{2}{*}{ Quetiapine* } & $12.5 \mathrm{mg}$ TID & \multirow{2}{*}{ PO } \\
\hline & 25mg HS SCH & \\
\hline Risperidone & $0.5 \mathrm{mg}$ BID PRN & PO, ODT \\
\hline \multirow{2}{*}{ Olanzapine } & $2.5 \mathrm{mg}$ BID & \multirow{2}{*}{ PO, ODT, IM } \\
\hline & $2.5 \mathrm{mg} \mathrm{HS}$ & \\
\hline Ziprasidone & 10mg BID PRN & PO, IM \\
\hline Aripiprazole & 5mg BID PRN & PO \\
\hline Valproic Acid & 125-250mg TID & PO, IV \\
\hline Melatonin* & 1-3mg HS & PO \\
\hline \multirow[t]{4}{*}{$\begin{array}{l}\text { *Quetiapine and Melatonin are some of the options for } \\
\text { sleep aid, to maintain circadian rhythm }\end{array}$} & BID=twice daily & $\begin{array}{c}\mathrm{PO}=\text { by mouth } \\
\mathrm{IV}=\text { intravenous } \\
\mathrm{IM}=\text { intramuscular } \\
\text { ODT=dissolvable }\end{array}$ \\
\hline & $\mathrm{TID}=$ three times daily $\mathrm{mg}=$ milligrams & \\
\hline & $\mathrm{HS}=$ at night $\mathrm{SCH}=$ scheduled & \\
\hline & PRN=as needed & \\
\hline
\end{tabular}




\section{Discussion}

This case report helps simplify the steps a provider should take in approaching Delirium to minimize all-cause mortality, for which FDA has issued a black box warning [1]. As Delirium can be seen in any hospital unit, all healthcare providers would benefit from this case report to learn how to approach delirium in an acute setting, to better serve our patients. Sure, psychiatry or neurology may be consulted at any time, however it is imperative to know the appropriate approach so the primary team, in any setting, can handle this presentation appropriately in an acute situation. The first approach should be nonpharmacological including environmental cues and reorientation with clock and calendar in the room, open window shades when patient is awake, more frequent family visits, and attempt to have the same caregiver as long as possible [1]. After exhausting nonpharmacological approaches, pharmacological approaches may be used, including low- dose antipsychotics, with a maximum daily dose of the lower limit of the therapeutic range of that antipsychotic [1]. Efforts to improve circadian rhythm are useful pharmacological methods to prevent delirium onset, and may be considered as a scheduled sleep aid (Table 1) upon hospitalization, particularly in the high risk population; geriatric patients, comorbid Major Neurocognitive Disorders, and various medical conditions that result in cognitive impairment. Providers must be mindful that delirium may last indefinitely, and that medications discussed in this case report are not to cure, rather to manage the symptoms [1].

\section{References}

1. Thom R, Levy-Carrick N, Phil M, David Silbersweig (2019) Delirium. Am J Psychiatry 176: 785-793.

2. Desk Reference to the Diagnostic Criteria From DSM-5. $5^{\text {th }}$ ed. American Psychiatric Association. Washington; 2013: pp. 292-295. 